$\overline{\text { REVIEW }}$-Current Perspective-

\title{
Fundamental Role of Heme Oxygenase in the Protection Against Ischemic Acute Renal Failure
}

\author{
Reiko Akagi ${ }^{1, *}$, Toru Takahashi ${ }^{2}$ and Shigeru Sassa ${ }^{3}$ \\ ${ }^{I}$ Department of Nutritional Science, Faculty of Health and Welfare Science, Okayama Prefectural University, \\ 111 Kuboki, Soja, Okayama 719-1197, Japan \\ ${ }^{2}$ Department of Anesthesiology and Resuscitology, Okayama University Medical School, 2-5-1 Shikata-cho, Okayama 700-8558, Japan \\ ${ }^{3}$ Laboratory of Biochemical Hematology, The Rockefeller University, New York, N.Y., 10021, USA
}

Received October 9, 2001

\begin{abstract}
Oxidative stress conditions such as oxidant stimuli, inflammation, exposure to xenobiotics and ionizing irradiation provoke cellular responses, principally involving transcriptional activation of genes encoding proteins that participate in the defense against oxidative tissue injuries. Excess of free heme, which is released from hemeproteins under these conditions, may constitute a major threat because it catalyzes the formation of reactive oxygen species. Exposure of mammalian cells to oxidative stimuli induces heme oxygenase-1 (HO-1), the rate-limiting enzyme in heme degradation, as well as the $32-\mathrm{kDa}$ heat shock protein. In various tissue injury systems, HO-1 induction has been shown to confer protection, while its abrogation has been shown to accelerate cellular injuries. In this review, recent findings concerning the role of HO-1 as a protective response against oxidative stress conditions are summarized, with a particular emphasis on its protective role in ischemic acute renal failure.
\end{abstract}

Keywords: Ischemic acute renal failure, Heme oxygenase, Tin, Heme, Oxidative stress

\section{Introduction}

Heme oxygenase (HO)-1 is the rate-limiting enzyme in the degradation of heme, which is catalyzed by a sequence of three enzymatic reactions; i.e., NADPH-cytochrome P450 reductase, HO-1 and biliverdin reductase (1). HO-1 converts heme to biliverdin IX $\alpha$, carbon monoxide and iron. Among the three $\mathrm{HO}$ isoforms known, HO-1 is highly inducible by a vast array of stimuli, including oxidative stress, heat shock, UV radiation, ischemia-reperfusion, heavy metals, bacterial lipopolysaccharide (LPS), cytokines, and nitric oxide, and its substrate, heme (1). HO-2 and $\mathrm{HO}-3$ are largely expressed in a constitutive fashion and probably function as heme binding molecules in normal cells $(2,3)$. Until recently, the heme-derived metabolites were thought to be useless waste or toxic products, but recent data suggest that they may have significant biological properties, such as anti-oxidative, anti-inflammatory, anti-apoptotic, signaling, and immune modulatory properties, and suppressing activities on adhesion molecule expression (4). The strong adaptive response of HO-1 to

*Corresponding author. FAX: 81-8669-94-2156

E-mail: akagirei@fhw.oka-pu.ac.jp various stimuli suggests an entirely new paradigm that HO-1 may play a significant role in the protection against inflammatory processes and oxidative tissue injuries.

Acute renal failure (ARF) is a frequent complication in critically ill patients in the intensive care unit (5). It may occur either as a part of the multiple organ dysfunction syndrome or as an isolated event. Accumulating data over the last two decades have indicated that ARF is characterized by a decrease in glomerular capillary permeability, back-leaks of glomerular filtrates, tubular obstruction and hemodynamic abnormalities (6). Reactive oxygen species (ROS) is thought to play an important role in the pathogenesis of ARF, and its formation can be greatly accelerated by the presence of iron. In certain models of ARF, the iron released from hemeproteins in the kidney is thought to be involved in ROS formation, thereby in the disease progression.

\section{HO-1 induction in the experimental model of ARF}

There are several experimental models of ARF, each with a unique feature. Each model has been used for assessing the role of HO-1 induction in the protection of the kidney from oxidative tissue injuries. 


\section{Glycerol-induced ARF}

The glycerol-induced ARF is most commonly used and is prepared by subcutaneous or intramuscular injection of hypertonic glycerol to rats (7). In this model, there are skeletal muscle injuries, termed "rhabdomyolysis", resulting in the release of myoglobin into plasma. It has been estimated that approximately one third of the patients with rhabdomyolysis develop ARF and that rhabdomyolysis may account for approximately $10 \%$ of all cases of ARF. It has been postulated that a large amount of heme released from myoglobin may be directly responsible for attendant lipid peroxidation (8).

In the kidney of rats treated with glycerol for $6 \mathrm{~h}, \mathrm{HO}-1$ mRNA was found to increase more than 50-fold, compared with that in untreated animals (9). The blockade of the increased $\mathrm{HO}$ activity by tin protoporphyrin (Sn-PP), a competitive inhibitor of $\mathrm{HO}$, significantly aggravated the renal injury in this model. Namely, serum creatinine concentrations increased more than twofold in Sn-PP treated animals compared with untreated controls, suggesting that renal injury was aggravated by the inhibition of $\mathrm{HO}$ activity. In contrast, induction of HO-1 by pretreatment of animals with hemoglobin prior to glycerol injection showed significant protection against the development of ARF (9). Thus, in this model, the exposure of the kidney to a single pretreatment of hemoglobin elicited induction of adaptive cellular responses that facilitated the clearance of inordinate amounts of cytotoxic free heme. These findings thus indicate that induction of $\mathrm{HO}-1$, which itself is a free heme-mediated process, also serves to clear an excess amount of free heme, ultimately resulting in a beneficial adaptive response.

\section{Cisplatin-induced toxic renal injury}

Cisplatin is a commonly used anticancer drug, but its use has to be often curtailed by its significant side effects, particularly nephrotoxicity. Cisplatin preferentially accumulates in the kidney and results in injuries in the proximal tubules (10). This unique effect of cisplatin was utilized to prepare an experimental model of toxic ARF. Both necrosis and apoptosis of renal tubular cells were observed in the cisplatin-induced ARF (11). Unlike in the glycerol-induced ARF, the oxidative stress in cisplatin-induced ARF appears to be unrelated to myoglobin, but rather may be due to the iron that is derived from microsomal cytochrome P450 in the kidney (12). It has been shown that HO-1 is induced in a time and dose-dependent fashion in the kidney after the administration of cisplatin, and administration of Sn-PP, which inhibits $\mathrm{HO}$ activity, aggravated the renal injury (13). HO-1 knockout mice have been shown to be highly sensitive to the toxic effect of cisplatin, and they developed more severe renal failure and have a higher degree of renal tubular apoptosis compared with the wild-type mice treated with cisplatin (11). In contrast, both pre-induction of HO-1 by hemin treatment and over-expression of the ho- 1 gene by gene targeting resulted in a significant amelioration of the cisplatin-induced renal injury (11).

\section{Ischemic ARF}

Ischemic ARF (IARF), which is due to the reperfusion injury of the kidney, accompanies acute tubular epithelial cell injury, and it is the major form of ARF of all ARF episodes in intensive care units. The IARF injury is thought to be due to ROS generated by reperfusion, which has been suggested to be a result of the rapid release of heme from microsomal cytochrome P450 (14). The reversibility of renal function in IARF critically depends on the length of the ischemic treatment prior to reperfusion, e.g., longer than 60-min ischemia, resulting in an irreversible renal damage (15). Rats following a unilateral nephrectomy, rats with the ligation of a contralateral renal artery, or rats with bilateral ligation followed by reperfusion, have been used as the experimental model of IARF. We found that both HO-1 mRNA and its enzyme activity were significantly increased in the reversible IARF model (16). Inhibition of HO activity by Sn-mesoporphyrin (Sn-MP) resulted both in a marked increase in intracellular heme content and in the aggravation of renal function in this model. HO-1 induction thus plays an important role in the protection against renal dysfunction due to oxidative damages caused by heme.

\section{HO inhibition worsens, while its induction alleviates IARF}

Certain metalloporphyrins have been shown to act as competitive inhibitors of HO activity (17). For example, Sn-PP or Sn-MP has been shown to be a clinically useful inhibitor of $\mathrm{HO}$ activity and is used for the treatment of neonatal jaundice (17). Sn-MP is a stronger inhibitor of $\mathrm{HO}$ activity than Sn-PP, presumably due to its better water solubility (17). In contrast to Sn-PP or Sn-MP, tin chloride $\left(\mathrm{SnCl}_{2}\right)$ treatment has been shown to potently induce HO-1, which is almost entirely restricted to the kidney (18). We examined the effect of $\mathrm{SnCl}_{2}$ or $\mathrm{Sn}-\mathrm{MP}$ in the kidney in rats with IARF as well as their effects on renal function as determined by morphological examination and by measurements of serum creatinine concentrations $(16,19)$. We have in fact confirmed a transient but strong induction of HO-1 mRNA in the kidney of rats following ischemia/reperfusion (16). $\mathrm{SnCl}_{2}$ treatment prior to ischemia/reperfusion, which is known to induce HO-1 specifically in the kidney, improved renal dysfunction, as judged by a decreased serum creatinine concentration (Fig. 1). In animals pretreated with $\mathrm{SnCl}_{2}$, the proximal tubular epithelial cells in the cortex were much less affected than $\mathrm{SnCl}_{2}$-untreated controls, and they were nearly normal (Fig. 1) (19). In 

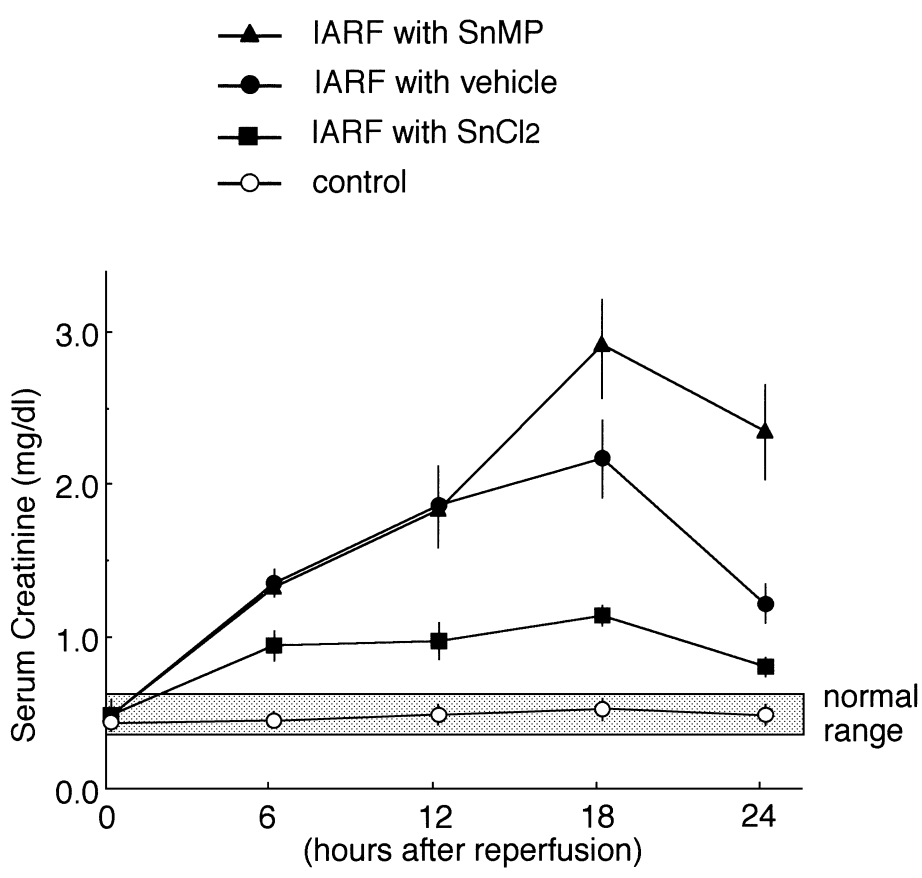

IARF with SnMP
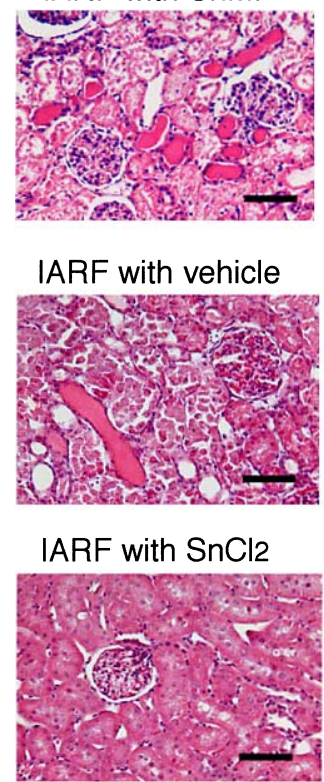

control

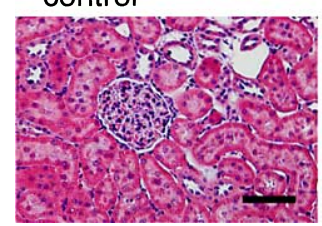

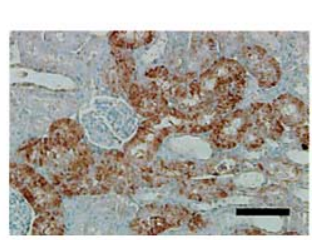
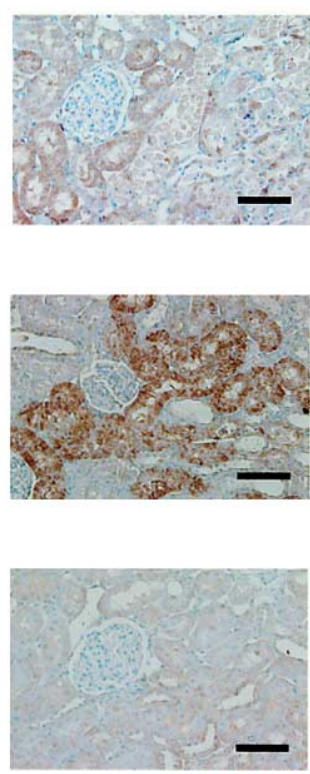

Fig. 1. Effect of $\mathrm{SnCl}_{2}$ or Sn-MP administration on serum creatinine and kidney tissue changes in rats with IARF. Rats were uninephrectomized and subjected to unilateral ischemia for $40 \mathrm{~min}$ to produce IARF. $\mathrm{SnCl}_{2}(10 \mathrm{mg} / 100 \mathrm{~g}$ body weight) was administered subcutaneously, and Sn-MP $(1 \mu \mathrm{mol} / \mathrm{kg}$ body weight $)$ was administered intravenously $24 \mathrm{~h}$ prior to the uninephrectomy. After the initiation of reperfusion, whole blood was collected for the determination of serum creatinine concentrations at $0,6,12,18$ and $24 \mathrm{~h}$. They are shown as the mean \pm S.E.M. $(n=6)$. Shown in the inset are histochemical changes in the renal cortex of rats following various treatments. Left: Kidney sections stained with hematoxylin and eosin. Extensive necrosis with cast formation in the proximal tubular cells was observed in IARF with vehicle treatment. It was aggravated in IARF with Sn-MP, in contrast to the relief from the injury in IARF with $\mathrm{SnCl}_{2}$ pretreatment. Right: Kidney sections stained immunohistochemically using anti-rat HO-1 as a primary antibody. After IARF, HO-1 protein was induced in tubular epithelial cells, while the induction became obvious in IARF with $\mathrm{SnCl}_{2}$ pretreatment. The bar represents $100 \mu \mathrm{m}$.

contrast, inhibition of $\mathrm{HO}$ activity by the administration of $\mathrm{Sn}$-MP to $\mathrm{SnCl}_{2}$-pretreated animals abolished the beneficial effect of the $\mathrm{SnCl}_{2}$ pretreatment on ischemic renal dysfunction, indicating the fundamental protective role of HO-1 in the renal epithelial cells against oxidative stresses (19).

Although HO-1 mRNA in the kidney of untreated rats was hardly detectable, it was markedly increased in the kidney following $\mathrm{SnCl}_{2}$ treatment, while it was barely detectable in the liver, lung, heart and small intestine. This result indicates that $\mathrm{HO}-1$ mRNA is induced by $\mathrm{SnCl}_{2}$ treatment in a highly kidney-specific manner (19). Following $\mathrm{SnCl}_{2}$ treatment, a marked elevation of renal HO-1 mRNA was observed, followed by increases in HO-1 protein expression and HO activity (19). HO-1 protein accumulated also specifically in the renal tubular epithelial cells, following $\mathrm{SnCl}_{2}$ treatment (Fig. 1). Since the renal tubular epithelial cells are the target cells in the renal ischemia/reperfusion injury, induction of HO-1 specifically in these cells indicates its critical association to the oxidative stress in these cells.

Ischemia/reperfusion was found to rapidly increase microsomal free heme concentrations in saline-treated rats, whereas $\mathrm{SnCl}_{2}$-pretreatment prevented it from occurring. Tin pretreatment also significantly attenuated the severity of ischemic renal injury. In contrast, when $\mathrm{SnCl}_{2}$-treated animals were treated with Sn-MP, microsomal heme concentrations were elevated and resulted in an aggravation of renal function (19). Thus, HO-1 induced by $\mathrm{SnCl}_{2}$ pretreatment effectively decreased the excess amount of toxic "free heme", resulting in the amelioration of the ischemic renal injury.

\section{Mechanisms of ho-1 gene induction in IARF}

Although the precise mechanism(s) of renal injury caused 
by ischemia/reperfusion remains elusive, ROS produced by reperfusion following ischemia appears to be one of the important mediators of tissue injury in IARF (6). It was found that there is a rapid increase in microsomal heme concentration, which is thought to be liberated from cytochrome P450 (12), and occurs in the kidney immediately after reperfusion (16). This form of heme is apparently not associated with apoproteins, since it acts as a potent pro-oxidant, leading to the generation of ROS and aggravates the ischemic renal injury. ROS also induces the breakdown of hemeproteins which further aggravates oxidative tissue damages by liberating more heme. Under these circumstances, ho- 1 gene expression is transcriptionally increased in various cell types (20). In contrast, HO-1 deficient embryonic fibroblasts are known to be hypersensitive to the cytotoxicity of both hemin and hydrogen peroxide (21).

Recent evidence points to the fact that a group of oxidative stress inducible genes is under the immediate transcrip- tional control of Nrf2-small Maf heterodimer-regulatory protein (Fig. 2). Nrf2 forms a heterodimer with a small Maf protein, interacts with an anti-oxidant responsive element (ARE), and induces transcription of a set of genes that encode anti-oxidant functions. Nrf2 thus regulates stress inducible protein genes via ARE (Fig. 2). For example, Nrf2-deficient cells have been shown to be hypersensitive to oxidative stresses (22). Various ROS-inducing agents increase the DNA binding activity of Nrf2 in the nucleus without influencing its mRNA level (23). In this manner, Nrf2 regulates a wide-range of metabolic responses to oxidative stress, which includes, among others, ho-1 (23). The ARE cognate sequence shares a high degree of homology to the consensus Maf recognition element (MARE) sequence, permitting AREs to be competitively bound by a number of bZip transcription factors in the Maf, Jun, Fos and Cap'n'Collar families (23) (Fig. 2). Because of their responsiveness to a wide variety of stress agents including oxidative stresses, the MAREs were also named

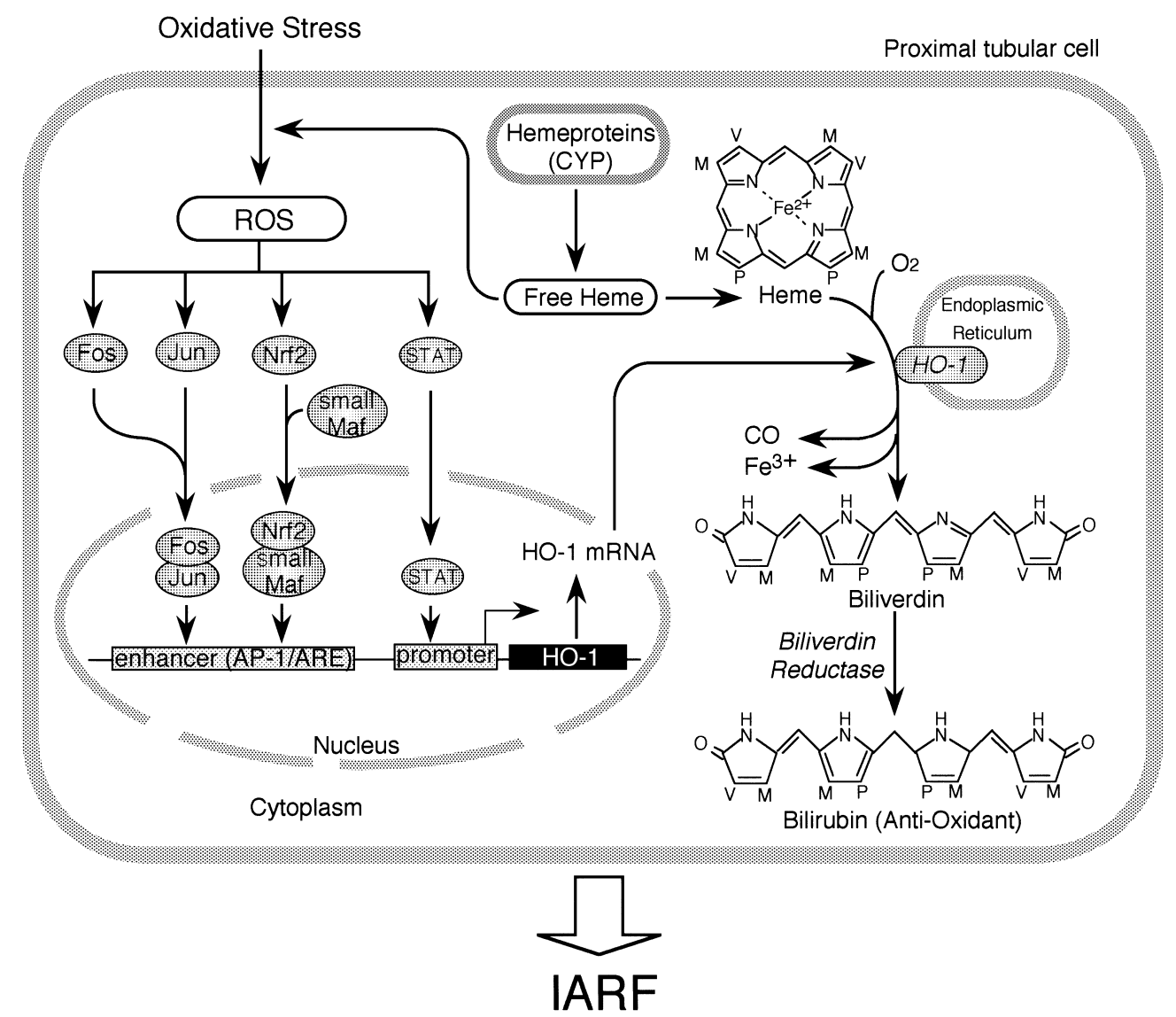

Fig. 2. Hypothetical mechanism of HO-1 induction in IARF. Oxidative stresses liberate Nrf2 from the cytoskeleton into the nucleus (32). Nrf2 then forms a heterodimer with a small Maf protein, interacts with ARE, and induces transcription of a set of genes that encode antioxidant activities (23), one of which is ho-1. Cytosolic free heme contributes to the generation of ROS, which in turn facilitates the formation of a Nrf2/small Maf heterodimer. Induction of HO-1 protein results in the removal of the pro-oxidant free heme, in the production of the anti-oxidant bile pigments, and in the formation of the anti-apoptotic CO. When the synthesis of ROS exceeds the level of detoxifying capacity of the renal proximal tubular cell, IARF ensues as the consequence of the reperfusion injury. CYP: cytochrome P450. 
stress-responsive elements (StREs) (24). Multiple StREs exist in ho- $l$ enhancers, and they have been shown to play important roles in the regulation of ho- 1 gene expression by various oxidative stresses, ROS, heavy metals and LPS (23). For example, the DNA binding activity of transcription factor AP-1 was inhibited when AP-1 was exposed to oxidative stress in vitro (25), which in turn may allow the competitive binding of other activating transcription factors, such as an Nrf2-small Maf heterodimer, to ARE and hence induce genes with protective properties against oxidative stress; e.g., ho-1 (23). While exposure of human MCF-7 cells to $\mathrm{CdCl}_{2}$, a potent inducer of HO-1, stimulates phosphorylation of ERK, JNK and p38 mitogen-activated kinases, an inhibitor of $\mathrm{p} 38$, or co-expression of a dominant-negative mutant of p38 $\alpha$, but not of ERK1, ERK2, JNK1 or JNK2, decreased HO-1 mRNA levels significantly (26). A dominant negative mutant of Nrf2 (a CNC-bZip member), but not of c-Jun or C/EBP $\beta$, inhibited Cd-mediated transactivation (26). These findings, also together with others, suggest that an oxidative stress induces ho- 1 gene expression via sequential activation of the p38 kinase pathway and Nrf2 (26).

\section{Metabolic consequences of HO-1 induction}

The immediate and specific adaptive response of HO-1 expression on the wide variety of oxidative stimuli suggests an important role of HO-1 in the protection against oxidative stress conditions, in addition to its key role in oxidative heme catabolism. The importance of HO-1 in the protection from inflammation is now amply documented in mice and humans deficient in HO-1 (21, 27). The absence of HO-1 results in an abnormally elevated serum heme concentrations (approx. $0.5 \mathrm{mM}$ ) and various oxidative and inflammatory complications $(21,27)$.

Free heme, largely liberated from hemeproteins under oxidative conditions, is highly lipophilic and will likely intercalate into the lipid bilayers in adjacent cells. Exposure of cells to heme is known to stimulate the expression of adhesion molecules ICAM-1, VCAM-1 and E-selectin on endothelial cells in vitro, probably through heme-mediated generation of ROS, which underscores reactive inflammatory changes (4). HO breaks down the pro-oxidant heme into three elements; i.e., iron, biliverdin IX $\alpha$ and carbon monoxide (CO). Iron, which is an oxidant, is directly sequestered and inactivated by co-induced ferritin (28). Biliverdin IX $\alpha$ is rapidly converted by biliverdin reductase to bilirubin IX $\alpha$, which is an anti-oxidant (29). CO produced from heme by HO can suppress apoptosis of endothelial cells via the activation of p38 MAPK (30). Thus, all these metabolites of the $\mathrm{HO}$ reaction act as a member of the protective response against oxidative stimuli and contribute to suppress a series of oxidative tissue damages that are associated with IARF.

\section{Conclusion}

In this review, recent evidence suggesting the fundamental role of HO-1 in anti-oxidative responses is summarized. Both inhibition of $\mathrm{HO}$ activity and suppression of ho-l gene expression lead to aggravation of oxidative tissue injuries. In contrast, exogenous administration of HO-1 by gene transfer confers significant protection in a rat model of inflammation or hemoglobin-heme toxicity (31). These findings suggest that tissue-specific ho- 1 expression, either by tissue-specific induction of HO-1 by chemicals or tissuespecific ho-1 gene overexpression, may hold therapeutic usefulness. Thus, tin salts, which have been simply thought to be toxic, may offer a new mode of treatment of IARF, because of their highly kidney-specific HO-1 inducing property.

\section{Acknowledgments}

This work was in part supported by grants from USPHS DK32890, and from Grant-in-Aid for Scientific Research (13671582) from the Ministry of Education, Culture, Sports, Science and Technology of Japan. We are grateful to Professor Dr. Masahisa Hirakawa (Okayama University Medical School) for his encouragement and support in this work.

\section{REFERENCES}

1 Shibahara S: Regulation of heme oxygenase gene expression. Semin Hematol 25, 370 - 376 (1988)

2 Maines MD: The heme oxygenase system: a regulator of second messenger gases. Annu Rev Pharmacol Toxicol 37, $517-554$ (1997)

3 McCoubrey WK Jr, Huang TJ and Maines MD: Isolation and characterization of a cDNA from the rat brain that encodes hemoprotein heme oxygenase-3. Eur J Biochem 247, 725 - 732 (1997)

4 Wagener FADTG, Eggert A, Boeman OC, Oyen WJG, Verhofstadt A, Abraham NG, Adema G, van Kooyk Y, de Witte T and Figdor CG: Heme is a potent inducer of inflammation in mice and is counteracted by heme oxygenase. Blood 98, 1802-1811 (2001)

5 Cole L, Bellomo R, Silvester W and Reeves JH: A prospective, multicenter study of the epidemiology, management, and outcome of severe acute renal failure in a "closed" ICU system. Am J Resp Crit Care Med 162, 191 - 196 (2000)

6 Kelly KJ and Molitoris BA: Acute renal failure in the new millennium: time to consider combination therapy. Sem Nephrol 20, 4 - 19 (2000)

7 Hostetter TH, Wilkes BM and Brenner BM: Renal circulatory and nephron function in experimental acute renal failure. In Acute Renal Failure, Edited by Brenner BM and Lazarus JM, pp 99-115, Saunders, Philadelphia (1983)

8 Holt SG and Moore KP: Pathogenesis and treatment of renal dysfunction in rhabdomyolysis. Intensive Care Med 27, $803-$ 811 (2001)

9 Nath KA, Balla G, Vercellotti GM, Balla J, Jacob HS, Levitt $\mathrm{MD}$ and Rosenberg ME: Induction of heme oxygenase is a rapid, protective response in rhabdomyolysis in the rat. J Clin Invest 90, $267-270$ (1992) 
10 Ries F and Klastersky J: Nephrotoxicity induced by cancer chemotherapy with special emphasis on cisplatin toxicity. Am J Kidney Dis 8, 368 - 379 (1986)

11 Shiraishi F, Curtis LM, Truong L, Poss K, Visner GA, Madsen K, Nick HS and Agarwal A: Heme oxygenase-1 gene ablation or expression modulates cisplatin-induced renal tubular apoptosis. Am J Physiol Renal Physiol 278, F726 - F736 (2000)

12 Baliga R, Zhang Z, Baliga M, Ueda N and Shah SV: Role of cytochrome P-450 as a source of catalytic iron in cisplatininduced nephrotoxicity. Kidney Int 54, 1562 - 1569 (1998)

13 Agarwal A, Balla J, Alam J, Croatt AJ and Nath KA: Induction of heme oxygenase in toxic renal injury: a protective role in cisplatin nephrotoxicity in the rat. Kidney Int 48, $1298-1307$ (1995)

14 Paller MS, Hoidal JR and Ferris TF: Oxygen free radicals in ischemic acute renal failure in the rat. J Clin Invest 74, 1156 1164 (1984)

15 Finn WF and Chevalier RL: Recovery from postischemic acute renal failure in the rat. Kidney Int 16, 113 - 123 (1979)

16 Shimizu H, Takahashi T, Suzuki T, Yamasaki A, Fujiwara T, Odaka Y, Hirakawa M, Fujita H and Akagi R: Protective effect of heme oxygenase induction in ischemic acute renal failure. Crit Care Med 28, 809 - 817 (2000)

17 Drummond GS: Control of heme metabolism by synthetic metalloporphyrins. Ann NY Acad Sci 514, 87 - 95 (1987)

18 Blumberg BS: Clinical significance of serum haptoglobins. In Hemoglobin. Its Precursors and Metabolites, Edited by Sunderman FW and Sunderman FW Jr, pp 318-324, JB Lippincott Co, Philadelphia (1964)

19 Toda N, Takahashi T, Mizobuchi S, Fujii H, Nakahira K, Takahashi S, Yamashita M, Morita K, Hirakawa M and Akagi $\mathrm{R}$ : Tin chloride pretreatment prevents the renal injury in rats with ischemic acute renal failure. Crit Care Med (in press)

20 Keyse SM and Tyrrell RM: Heme oxygenase is the major 32-kDa stress protein induced in human skin fibroblasts by UVA radiation, hydrogen peroxide, and sodium arsenite. Proc Natl Acad Sci USA 86, 99 - 103 (1989)

21 Poss KD and Tonegawa S: Reduced stress defense in heme oxygenase 1-deficient cells. Proc Natl Acad Sci USA 94, 10925 - 10930 (1997)

22 Itoh K, Chiba T, Takahashi S, Ishii T, Igarashi K, Katoh Y, Oyake T, Hayashi N, Satoh K, Hatayama I, Yamamoto M and
Nabeshima Y: An Nrf2/small Maf heterodimer mediates the induction of phase II detoxifying enzyme genes through antioxidant response elements. Biochem Biophys Res Commun 236, 313 - 322 (1997)

23 Ishii T, Itoh $\mathrm{K}$, Takahashi $\mathrm{S}$, Sato $\mathrm{H}$, Yanagawa $\mathrm{T}$, Katoh $\mathrm{Y}$, Bannai S and Yamamoto M: Transcription factor Nrf2 coordinately regulates a group of oxidative stress-inducible genes in macrophages. J Biol Chem 275, 16023 - 16029 (2000)

24 Alam J, Camhi S and Choi AM: Identification of a second region upstream of the mouse heme oxygenase- 1 gene that functions as a basal level and inducer- dependent transcription enhancer. J Biol Chem 270, 11977 - 11984 (1995)

25 Abate C, Patel L, Rauscher FJ and Curran T: Redox regulation of fos and jun DNA-binding activity in vitro. Science 249, $1157-1161$ (1990)

26 Alam J, Wicks C, Stewart D, Gong P, Touchard C, Otterbein S, Choi, AM, Burow ME and Tou J: Mechanism of heme oxygenase-1 gene activation by cadmium in MCF-7 mammary epithelial cells. Role of p38 kinase and $\mathrm{Nrf} 2$ transcription factor. J Biol Chem 275, 27694 - 27702 (2000)

27 Yachie A, Niida Y, Wada T, Igarashi N, Kaneda H, Toma T, Ohta K, Kasahara Y and Koizumi S: Oxidative stress causes enhanced endothelial cell injury in human heme oxygenase-1 deficiency. J Clin Invest 103, 129 - 135 (1999)

28 Vogt BA, Alam J, Croatt AJ, Vercellotti GM and Nath KA: Acquired resistance to acute oxidative stress. Possible role of heme oxygenase and ferritin. Lab Invest 72, $474-483$ (1995)

29 Stocker R, Yamamoto Y, McDonagh AF, Glazer AN and Ames $\mathrm{BN}$ : Bilirubin is an antioxidant of possible physiological significance. Science 235, 1043 - 1046 (1987)

30 Brouard S, Otterbein LE, Anrather J, Tobiasch E, Bach FH, Choi AM and Soares MP: Carbon monoxide generated by heme oxygenase 1 suppresses endothelial cell apoptosis. J Exp Med 192, 1015 - 1026 (2000)

31 Yang L, Quan S and Abraham NG: Retrovirus-mediated HO gene transfer into endothelial cells protects against oxidantinduced injury. Am J Physiol 277, L127 - L133 (1999)

32 Itoh K, Wakabayashi N, Katoh Y, Ishii T, Igarashi K, Engel JD and Yamamoto M: Keap1 represses nuclear activation of antioxidant responsive elements by Nrf2 through binding to the amino-terminal Neh2 domain. Genes Dev 13, 76 - 86 (1999) 